\title{
Data Downloading on the Sparse Coverage-Based Wireless Networks
}

\author{
Helal Chowdhury, Janne Lehtomäki, Juha-Pekka Mäkelä, and Sastri Kota
}

Centre for Wireless Communications, University of Oulu, 90014 Oulu, Finland

Correspondence should be addressed to Helal Chowdhury, fougi5@yahoo.com

Received 26 February 2010; Accepted 30 April 2010

Academic Editor: Dharma Agrawal

Copyright (๑) 2010 Helal Chowdhury et al. This is an open access article distributed under the Creative Commons Attribution License, which permits unrestricted use, distribution, and reproduction in any medium, provided the original work is properly cited.

\begin{abstract}
Infostation, hotspot, and drive-thru internet are examples of sparse coverage-based wireless networks. These wireless communication networks provide low-cost, delay insensitive high data rate services intermittently with discontinuous coverage. Radio propagation parameters, velocity of the user, distance between the user, and access point are the key factors that affect the throughput and the amount of information downloaded from such sparse coverage-based wireless networks. To evaluate the performance of such wireless communication networks analytically the impact of above mentioned factors can be modeled with simplified relationship model such as received signal strength versus distance or signal to noise ratio versus throughput. In the paper, we exploit the relationship between throughput and distance and develop two throughput distance relationship models to evaluate the performance of multirate wireless networks. These two throughput distance relationship models are used in calculation of average throughput as well as downloaded file size. Numerical values are presented for the IEEE 802.11n standard. The numerical results verify that the new proposed technique can be used as an alternative to the simulations to evaluate the performance of sparse coverage-based wireless networks.
\end{abstract}

\section{Introduction}

Data download on the move can be seen as a promising application for the next generation wireless communication systems [1]. Infostation is one example of such paradigm of wireless communication systems for delivering information services to a mobile user. The key assumption for such wireless communication systems is that the coverage is sparse, as a result, as long as the mobile user is in the coverage area of Infostation the user may download information to the mobile terminal storage for later usage. The retrieved information can be a video file, a piece of music, a weather report, and so forth. Although the concept of Infostation is not new, there has recently been a renewed interest for such model for information distribution [2-6]. Hotspot [7], drive-thru internet [8], and roadside infrastructures [6] are examples of sparse coverage-based wireless networks. Even though the naming varies in terms of functionalities they share major commonalities with Infostation. The overall coverage of these wireless communication systems is considered to be sparse [8]. High data rate and support for mobility make IEEE802.11-based WiFi wireless local-area networks (WLAN) a promising candidate to be used as an underlying technology when implementing the aforementioned sparse coverage wireless communication systems. Measurementbased performance analysis of IEEE802.11b/g-based systems for static and nomadic users as well as in case of outdoor drive-through scenario also has been carried out extensively in the past and reported in [5, 8-13]. However, very few papers addressed the issue of performance analysis of WLAN-based system specially data download on move scenario $[1,14]$. In contrast to $[1,14]$, in this work we will provide the methodology to evaluate the performance of IEEE802.11n-based network in the context of data downloading in walk through scenario by using polynomial as well as two-slope throughput-distance model.

The throughput is affected by the communication environment and related channel conditions. The effecting factors include the distance between the transceivers, fading state of the channel, noise, and interference. The effect of 
all these parameters to the obtained transmission throughput can approximately be described with the throughputdistance relationship model [15].

In this work we enhance the previous work and results by providing mathematical framework utilizing throughputdistance models to evaluate the performance of sparse coverage-based networks. The results and methodology presented provide the insightful knowledge of deployment related issues of sparse coverage network which can be used before it is deployed in reality.

\section{Mathematical Framework}

A typical scenario of data downloading is shown in Figure 1, where a user with mobile terminal (MT) enters and exits the coverage area from arbitrary directions and downloads data. When user passes through the coverage of the access point (AP) it observes several data-rates. As it is mentioned, the closer the user is to the AP, the higher data-rate it receives from it.

We assume that the AP is at coordinates $(0,0)$ and that the user enters the coverage at point $A^{\prime}$, with coordinates $\left(x_{a}^{\prime}, y_{a}^{\prime}\right)$ known as entering point and exits the coverage at point $B^{\prime}$, with coordinates $\left(x_{b}^{\prime}, y_{b}^{\prime}\right)$ known as exit point, as shown in Figure 1. The entrance angle is denoted with $\alpha_{1}$, and the exit angle is denoted with $\alpha_{2}$. The angle between entrance point and exit point is called traveling angle which is denoted as $\theta$.

Assuming constant velocity, the path that the user travels can be simply expressed with

$$
(x(t), y(t))=\left(\left(x_{b}^{\prime}-x_{a}^{\prime}\right) t+x_{a}^{\prime},\left(y_{b}^{\prime}-y_{a}^{\prime}\right) t+y_{a}^{\prime}\right)
$$

where the parameter $t$ varies from zero (corresponding to the entrance point) to one (the exit point). Let us characterize the throughput as a function of the distance, that is, the throughput at distance $r$ is $S(r)$. Now, we can find the average throughput of the user (while it passes through the coverage) with

$$
S_{\mathrm{av}}=\int_{t=0}^{1} S\left(\sqrt{\left[\left(x_{b}^{\prime}-x_{a}^{\prime}\right) t+x_{a}^{\prime}\right]^{2}+\left[\left(y_{b}^{\prime}-y_{a}^{\prime}\right) t+y_{a}^{\prime}\right]^{2}}\right) d t .
$$

However, the calculation of (2) for arbitrary entrance and exit points is cumbersome. In this paper we provide a novel rotational technique where any inclined traveling path as in Figure 1 can be presented into equivalent horizontal path as shown in Figure 2. In this case calculation of average throughput over inclined path $\left(A^{\prime} B^{\prime}\right)$ by using (2) will be the same as the calculation of average throughput over horizontal path $(A B)$. Depending on the difference between the entrance and exit angle the rotation of traveling angle $\theta$ can be expressed as

$$
\theta= \begin{cases}\left|\alpha_{1}-\alpha_{2}\right| & \left|\alpha_{1}-\alpha_{2}\right| \leq 180^{\circ} \\ 360^{\circ}-\left|\alpha_{1}-\alpha_{2}\right| & \left|\alpha_{1}-\alpha_{2}\right|>180^{\circ} .\end{cases}
$$

It should be noticed that using (3) the maximum range of traveling angle can be limited to in the range of $0^{\circ}$ to

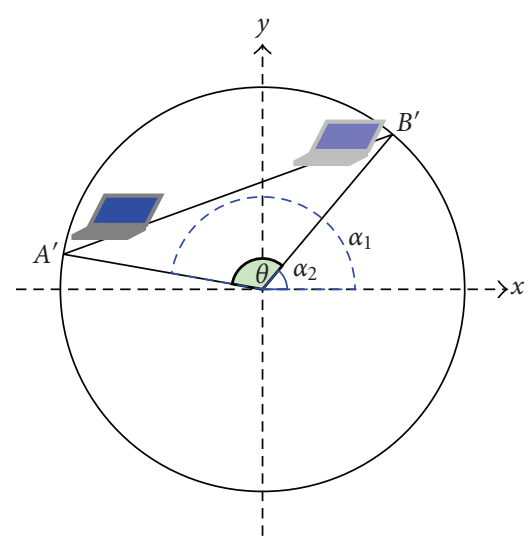

FIgURE 1: Scenario of datadownload on go.

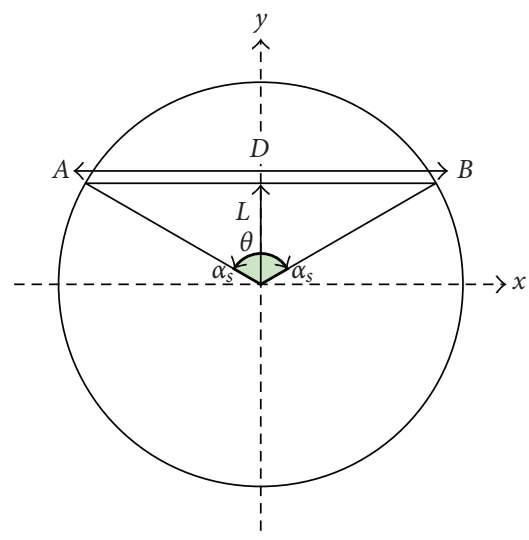

FIGURE 2: Entrance and exit points after rotation to achieve horizontal traveling path.

$180^{\circ}$ instead of $0^{\circ} 360^{\circ}$ for calculation of average throughput over simplified horizontal path. The calculation of average throughput over simplified horizontal path is given below.

After rotation, we get new entrance point $A$ and exit point $B$, as shown in Figure 2. In the Figure it is also shown that the minimum distance between mobile user and the AP is $L$, which for a particular traveling angle $\theta$ is $L=$ $R \cos (\theta / 2)$, where $R$ is the coverage radius. The traveling distance $D=2 R \sin (\theta / 2)$. However it should be noted that the traveling distance is equal before and after rotation (e.g., $\left.D=A^{\prime} B^{\prime}=A B\right)$ that is the traveling angle $\theta$ is not affected by the rotation. Finally average throughput over simplified horizontal path can be expressed as

$$
S_{\mathrm{av}}=\int_{t=0}^{1} S\left(\sqrt{[x(2 t-1)]^{2}+y^{2}}\right) d t
$$

where $x=D / 2=R \sin (\theta / 2)$ and $y=L$. It is easily seen that (4) leads to much simplified integration in compare to (2).

Taking velocity $v$ into account, the dwelling time of the mobile user can be found with $t_{\text {dwell }}=D / v=2 x / v$. Now by simply multiplying the dwell time with the average throughput observed during the path, we get that size of the 
transferred information which is termed as file size can be expressed as

$$
I_{t}(\theta)=\frac{2 x}{v} \int_{t=0}^{1} S\left(\sqrt{[x(2 t-1)]^{2}+y^{2}}\right) d t .
$$

\section{Throughput Distance Models}

Utilizing our methodology to evaluate the performance of sparse-based coverage network we need to develop first throughput-distance model. Throughput distance model can either be measurement-based or theoretical-based throughput-distance model. In this work, first we derived theoretical throughput-distance model which we call it quantized throughput-distance model and then approximated it by polynomial and two-slope throughput-distance model for further analysis. We have considered IEEE 802.11n technology which uses multiplexing (SDM) technique to increase the data rate [16]. In SDM, data rate is increased as the number of spatial stream (NSS) is increased. We consider that to handle more than one NSS number of transmit and receive antennas is also increased according to the number of NSS. We consider each stream is identical and independent. As a result, in ideal case for NSS $=2$ the data rate is just doubled in compare to NSS $=1$. Data rate associated with NSS is shown in Table 1 [17]. The purpose of this consideration is to treat certain aspects in general way, while modeling other aspects in more great details. The data rate using different modulation and coding (MCS) for NSS $=1$ and NSS $=2$ at $20 \mathrm{MHz}$ channel for $800 \mathrm{~ns}$ guard interval and minimum receiver sensitivity for nonspace time block coding (STBC) mode is shown in Table 1 [17]. Utilizing these information we will first derive the throughput-distance model for single stream $(1 \times 1)$ case. Upon getting the throughput-distance model for $1 \times 1$ case throughput-distance relationship for $2 \times 2$ case can be derive by just doubling data rate of $1 \times 1$ and use the same coverage range which has been achieved for $1 \times 1$ case. The calculation of throughput-distance model is given below. Distance-based pass-loss model for $1 \times 1$ antenna case can be expressed as [15]

$$
L_{p}[\mathrm{~dB}]=L_{0}[\mathrm{~dB}]+10 \alpha \log _{10}(d),
$$

where $\alpha$ is the path loss exponent. $L_{p}$ and $L_{0}$ are the total path-loss and path-loss at a distance of one meter respectively. $d$ is the distance between transmitter and receiver and expressed in meter. In terms of powers, $L_{p}$ and $L_{0}$ can be expressed as $L_{p}[\mathrm{~dB}]=p_{t}[\mathrm{dBm}]-p_{r}[\mathrm{dBm}]$ and $L_{0}[\mathrm{~dB}]=10 \log _{10}\left(p_{t}[\mathrm{~W}]\right)-10 \log _{10}\left(p_{0}[\mathrm{~W}]\right)$ where $p_{t}, p_{r}$, and $p_{0}$ are the transmit power, receive power, and power at reference distance (1 meter), respectively. Assuming transmitter and receive antenna gain 1 and using receiver sensitivity from Table 1 the distance associated for maximum achievable data rate can be calculated as

$$
d=10^{\left(L_{p}-L_{0}\right) / 10 \alpha} .
$$

Finally the related computed distance in (7) and maximum achievable data rate for specified receive power is achieved.

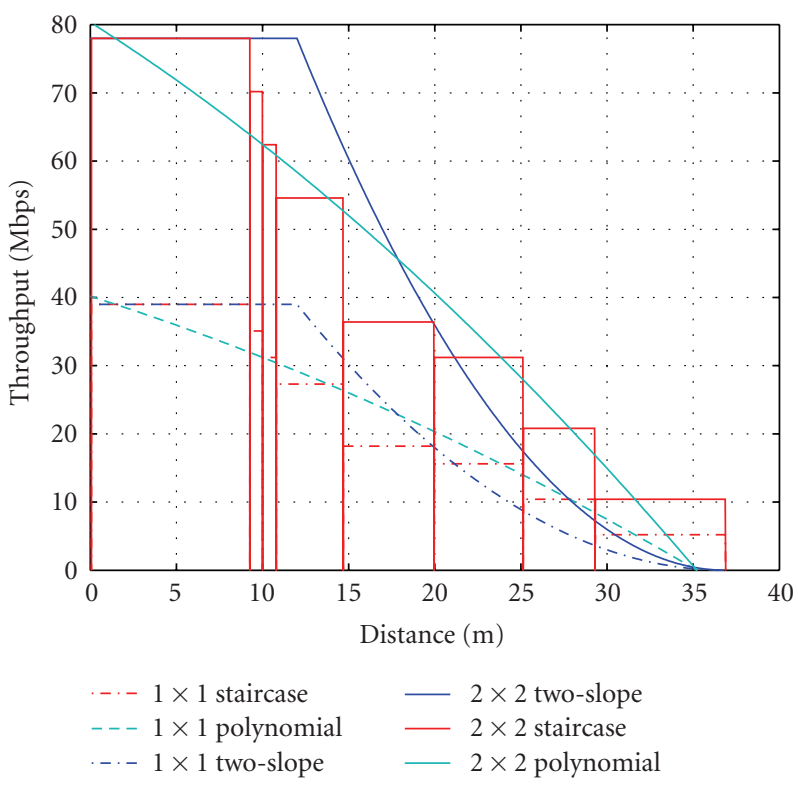

FIgURE 3: Theoretical Quantized and its approximated polynomial and two-slope throughput distance models.

However, it should be noted that the data rate which has been specified in Table 1 is the physical data rate hence to achieve user level data rate we exclude overheads from the physical data rate. It is known that as the data rate increases the the percentage of overheads also increases. In this respect, we classify the available data rate into three groups: higher data rate (65 Mbps, $58.50 \mathrm{Mbps}, 52.0 \mathrm{Mbps}$ ), middle data rate $(39 \mathrm{Mbps}, 26 \mathrm{Mbps})$ and lower data rate (19.50 Mbps, 13.0 Mbps, 6.5 Mbps). We exclude 40\%, 30\%, $20 \%$ overheads from the higher, middle and lower data rate respectively [18]. Finally user level data rate has been plotted against distance and named as throughput-distance model. The derived throughput-distance relationship model for $1 \times 1$ and $2 \times 2$ antenna case is shown in Figure 3 .

3.1. Model I: Polynomial. In polynomial model a secondorder polynomial is used to approximate the theoretical relationship between throughput and distance. In this case we ran a MATLAB basic fitting tool to fit the theoretical throughput-distance to polynomial throughput distance model. This basic fitting tool uses least square method (LS). The approximated second order polynomial throughputdistance relationship can be expressed as

$$
S(r)= \begin{cases}a r^{2}+b r+c, & 0<r<R, \\ 0, & \text { otherwise. }\end{cases}
$$

Substituting (8) into (4) and performing the integration the average throughput is given by

$$
S_{\mathrm{av}}(r)=a\left(\frac{x^{2}}{3}+y^{2}\right)+\frac{b}{4 x}\left(2 R x-y^{2} \log \frac{(-x+R)}{(x+R)}\right)+c,
$$


TABLE 1: Physical modes and corresponding throughput, NSS, receiver sensitivity [17].

\begin{tabular}{lccc}
\hline Modulation & NSS $=1$, Datarate $[\mathrm{Mbps}]$ & NSS = 2, Datarate $[\mathrm{Mbps}]$ & Receiver Sensitivity $[\mathrm{dBm}]$ \\
\hline BPSK $(1 / 2)$ & 6.5 & 13.0 & -82 \\
QPSK $(1 / 2)$ & 13.0 & 26.0 & -79 \\
QPSK (3/4) & 19.5 & 39.0 & -77 \\
16QAM (1/2) & 26.0 & 52.0 & -74 \\
16QAM (3/4) & 39.0 & 78.0 & -70 \\
64QAM (2/3) & 52.0 & 104.0 & -66 \\
64QAM (3/4) & 58.5 & 117.0 & -65 \\
64QAM (5/6) & 65.0 & 130.0 & -64 \\
\hline
\end{tabular}

which can be expressed in terms of the traveling angle $\theta$ by using $x=R \sin (\theta / 2)$ and $y=R \cos (\theta / 2)$. For the average throughput we get

$$
\begin{aligned}
S_{\mathrm{av}}(\theta)= & a R^{2}\left(1-\frac{2}{3} \sin ^{2}\left(\frac{\theta}{2}\right)\right) \\
& +b R\left(\frac{1}{2}+\frac{\cos ^{2}(\theta / 2)}{4 \sin (\theta / 2)}\right) \\
& \times \log \left(\frac{1+\sin (\theta / 2)}{1-\sin (\theta / 2)}\right)+c .
\end{aligned}
$$

Similarly, the file size can be expressed with

$$
\begin{aligned}
I_{t}(\theta)= & \frac{2 a R^{3}}{v} \sin \left(\frac{\theta}{2}\right) \\
& +\frac{b R^{2}}{2 v}\left(2 \sin \left(\frac{\theta}{2}\right)+\cos ^{2}\left(\frac{\theta}{2}\right) \log \frac{1+\sin (\theta / 2)}{1-\sin (\theta / 2)}\right) \\
& +\frac{2 R \sin (\theta / 2)}{v} c .
\end{aligned}
$$

3.2. Model II: Two-Slope. In our two slopes model, two cocentric circles are used to represent the different segments of traveling path of a mobile user. The inner tier is used to represent the saturation level and outer tier is used to represent distance decreasing function. The coverage range of outer tier and inner tier are represented as $R$ and $r_{\text {in }}$, respectively. $r$ is the instantaneous distance between mobile user and AP. Our proposed two-slope model can be expressed as

$$
S(r)= \begin{cases}S_{\max }, & 0<r<r_{\mathrm{in}}, \\ k(r-R)^{2}, & r_{\text {in }}<r<R,\end{cases}
$$

where $S_{\max }$ is the maximum throughput. The value of $k$ can be determined at maximum value of distance decreasing function which can be expressed as

$$
k=\frac{S_{\max }}{\left(r_{\text {in }}-R\right)^{2}} .
$$

When the user passes through the outer tier in that case average throughput observed by the user can be expressed as

$$
S_{o}(r)=k\left(\frac{R y^{2}}{x} \log \left(\frac{-x+R}{y}\right)+\frac{1}{3} x^{2}+y^{2}\right) .
$$

In terms of traveling angle $\theta(14)$ can be written as

$$
\begin{gathered}
S_{o}(\theta)=k R^{2}\left(\frac{\cos ^{2}(\theta / 2)}{\sin (\theta / 2)} \log \left(\frac{-\sin (\theta / 2)+1}{\cos (\theta / 2)+1}\right)\right. \\
\left.+1-\frac{2}{3} \sin ^{2}\left(\frac{\theta}{2}\right)\right) .
\end{gathered}
$$

When $y \leq R_{\text {in }}$ the user passes through both inner tier and outer tier the outer tier segment throughput can be expressed as

$$
\begin{aligned}
S_{\mathrm{oi}}(r)=\frac{\mathrm{k}}{\left(x-x_{c}\right)}( & -R y^{2} \log \left(\frac{x_{c}+r_{\mathrm{in}}}{x+R}\right)-x r_{\mathrm{in}} R-\frac{1}{3}\left(x^{3}-x_{c}^{3}\right) \\
& \left.-y^{2} x+x\left(y^{2}+R^{2}\right)\right)
\end{aligned}
$$

where $x_{c}$ is the $x$ component of the switching point. Switching point is the point when mobile user switches from outer tier to inner tier or vise versa. In terms of traveling angle $\theta(16)$ can be written as

$$
\begin{aligned}
& S_{\mathrm{oi}}(\theta)=\frac{k}{(x-R \sin (\theta / 2))} \\
& \times\left(R ^ { 2 } \operatorname { c o s } ^ { 2 } ( \frac { \theta } { 2 } ) \left(x-R \log \left(\frac{x_{c}+r_{\text {in }}}{R \sin (\theta / 2)+R}\right)\right.\right. \\
& -R \sin \left(\frac{\theta}{2}\right)-x r_{\text {in }} R \\
& \left.\left.-\frac{1}{3}\left(R^{3} \sin ^{3}\left(\frac{\theta}{2}\right)-x_{c}^{3}\right)+x R^{2}\right)\right) \text {. }
\end{aligned}
$$

Finally in calculation of two-slope throughput distance relation model case when the user passes through the outer circle the average throughput can be calculated by using (14) or (15), on the other hand when the user passes both through 
TAble 2: Simulation Parameters.

\begin{tabular}{ll}
\hline Parameter & Value \\
\hline Transmit power & $15 \mathrm{dBm}$ \\
Pathloss Exponent & 3 \\
No. of transmitter and receiver & $n=1,2$ \\
antennas & $a=-0.009 n$, \\
& $b=-0.8 n$, \\
Polynomial Coefficients & $c=40 n$ \\
$R_{\text {in }}$ & $12 \mathrm{~m}$ \\
$R_{\max }$ & $35.18 \mathrm{~m}$ \\
NSS & 1,2 \\
$T_{\max }$ & 78 \\
Carrier frequency & $2.4 \mathrm{GHz}$ \\
\hline
\end{tabular}

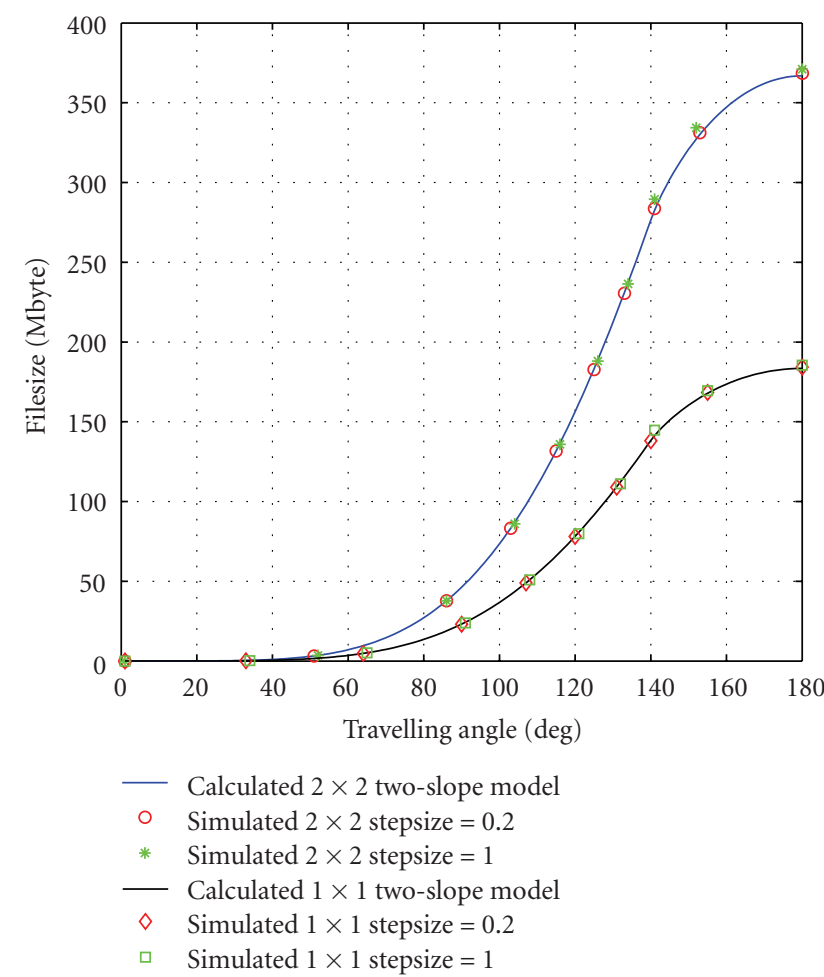

Figure 4: Comparison between simulation and theoretically computed results for two-slope throughput-distance model.

the outer circle and inner circle the average throughput can be calculated as

$$
S_{\mathrm{av}}=\frac{S_{\max } x_{c}+S_{o i}\left(x-x_{c}\right)}{x} .
$$

\section{Results and Discussion}

In this section throughput performance of sparse coveragebased wireless communication network evaluated using throughput distance models presented in Section 3 and

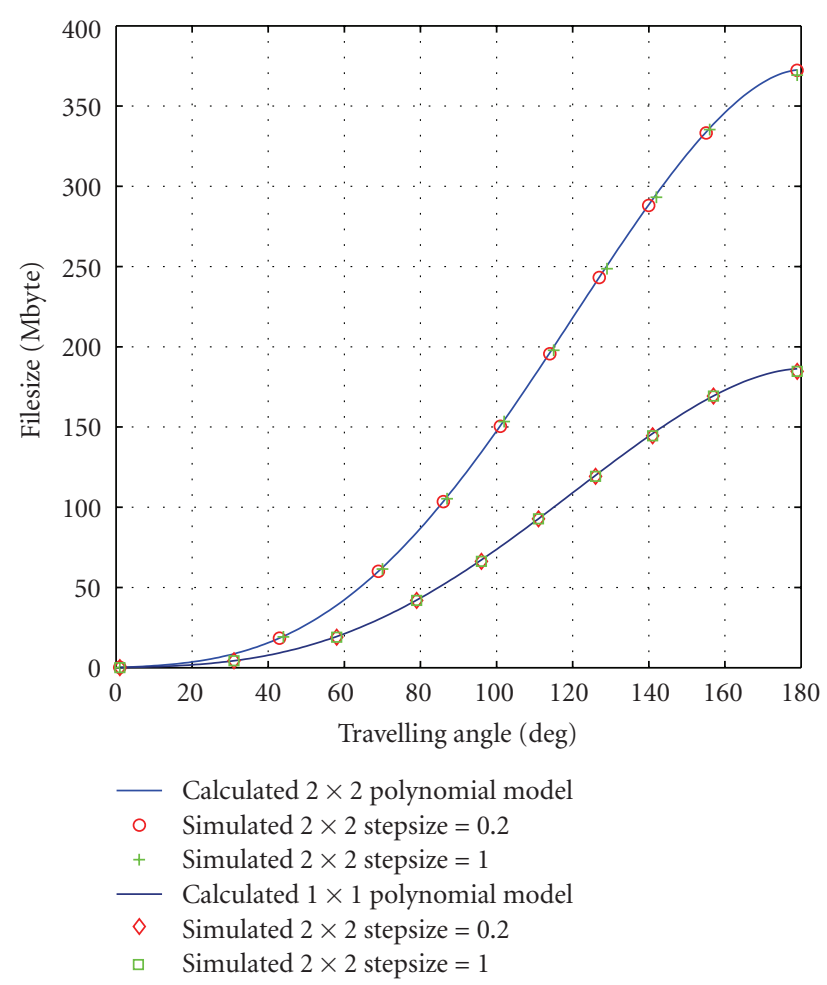

FIGURE 5: Comparison between simulation and theoretically computed results for Polynomial throughput-distance model.

parameters presented in Table 2. Figure 4 shows the comparison between the results obtained from simulation and result obtained using derived formulas for two-slope throughputdistance model. It is seen that theory and simulation result agree very well. In comparison, file size is used as performance metric. In simulation environment mobile user will move from enter point to exit point at constant speed along traveling distance with certain step size. In this case we have chosen two different step sizes. First we simulate for stepsize $=0.2 \mathrm{~m}$ and then for step size $=0.5 \mathrm{~m}$. In both cases simulation results agree with the result obtained using derived formula. Figure 5 shows the comparison between the results obtained from simulation and result obtained using derived formulas for polynomial throughput-distance model. In polynomial case it is also seen that the results obtained from simulation and calculated are perfectly matched.

Figure 6 shows the comparison of received file size at the coverage of IEEE $802.11 \mathrm{n}$ standard WLAN technology by a mobile user. Performance is evaluated by using two throughput distance relationship models and compared with quantized throughput-distance relationship model. In case of $2 \times 2$ antenna case it can be seen that the theoretical and its approximated polynomial and two-slope model the maximum file size that can be downloaded is 350 Mbyte. On the other hand, in case of $1 \times 1$ antenna case the maximum file size that can be downloaded is $175 \mathrm{Mbyte}$. It is also seen that higher the traveling angle higher the file size downloaded. Furthermore, it can be seen that according to our fitting 


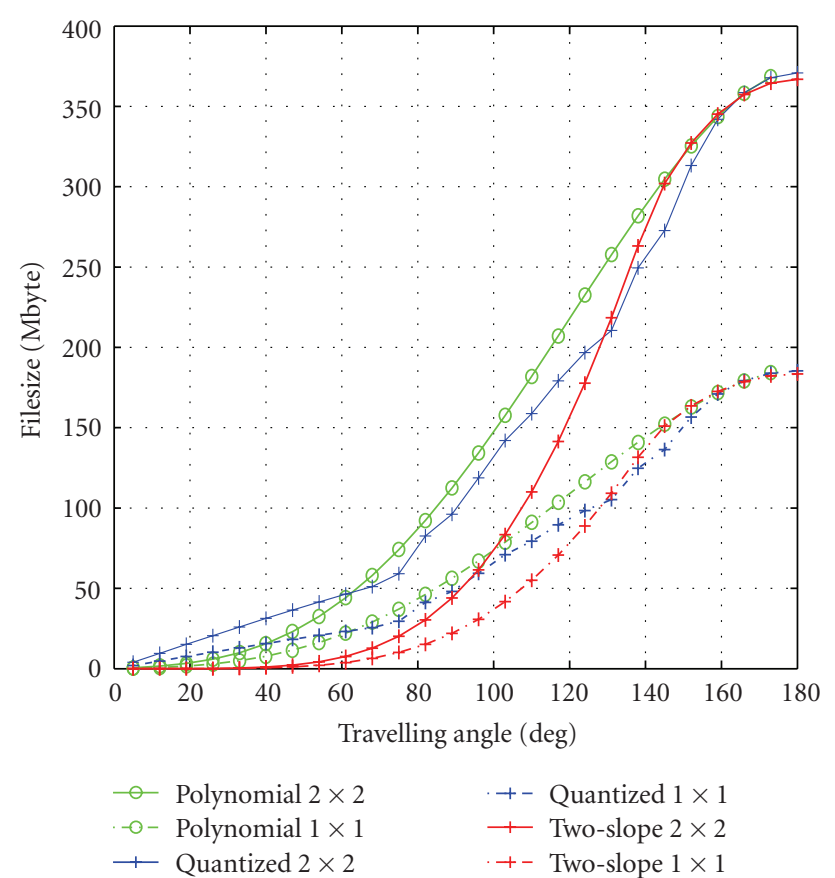

Figure 6: Comparison between theoretical and two proposed models.

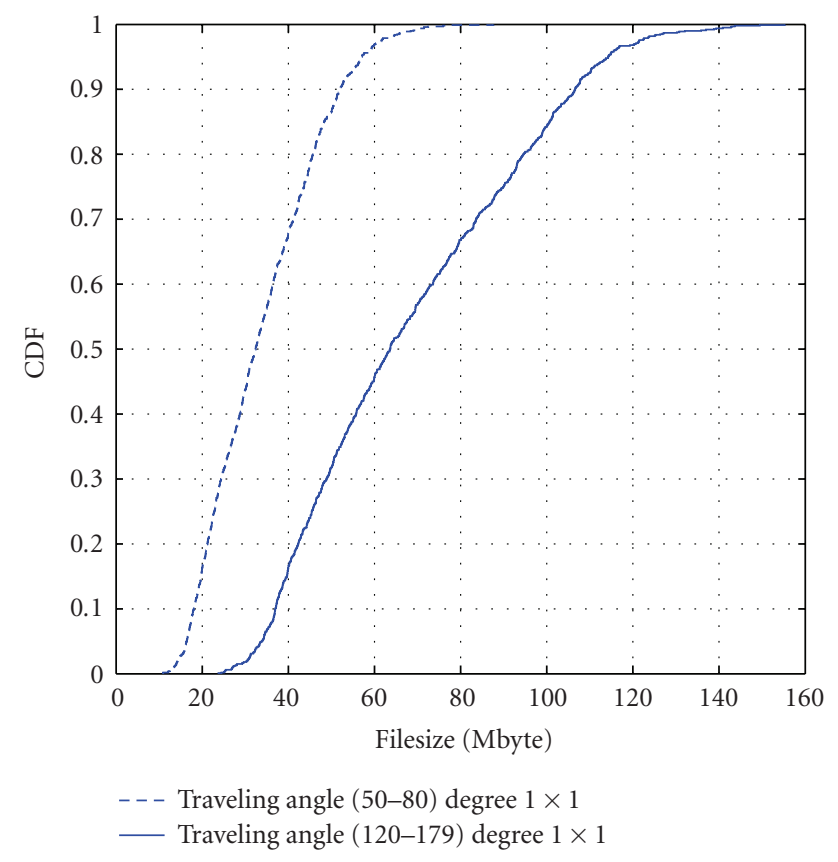

FIGURE 7: CDF of data download when velocity of user changes time to time along the traveling distance.

criteria approximation of quantized model with polynomial and two-slope model fitted well at the higher traveling angle. Figure 7 shows the CDF of downloaded file size while the user moves through the near or far corridor in $1 \times 1$ antenna case. Near corridor we mean the corridor which is close to the AP and far corridor are far from the AP. The length of the traveling distances of the near corridor will be much higher than far corridor. The length of traveling distance is controlled by the traveling angles. In such cases we assigned traveling angles between $\left(120^{\circ}-179^{\circ}\right)$ for near corridor and $50^{\circ}-80^{\circ}$ for the far corridor which will provide traveling distances for near corridor in between (117.71-135.91) $\mathrm{m}$ and (57-87.36) $\mathrm{m}$ for far corridor. When the user moves through any of the corridor the velocity will change time to time. The traveling distance has been divided into number of segments and in each segment velocity is assigned which is uniformly distributed between (1-3) m/s. It can been seen that there is very big difference in terms of file sizes between near and far corridor. With 50\% probability the downloaded file size is less than 30 Mbyte for far corridor and 64 Mbyte for near corridor. It is obvious that if the mobile user passes through the near corridor it receives much higher data rate than if the mobile user passes through far corridor, it reflects in the result as well in case of downloaded file size. Moreover, the chance to get more than 40 Mbyte for far corridor is 30\% which is $78 \%$ for near corridor.

\section{Conclusion}

In this work, we exploit the relationship between throughput and distance to evaluate the performance of sparse coveragebased wireless networks. The main contribution to this approach is to develop quantitative models to study the performance of sparse coverage-based wireless network by using theoretical-based throughput distance models. Our derived average throughput quantitative model is used to calculate file size that can be downloaded while mobile crosses the sparse coverage-based wireless networks. From our observation we can say that theoretical quantized throughput distance model and its approximated polynomial and two-slope model provide the upper bound of achievable average throughput which ultimately provides upper bound of downloaded file size. Finally, we argue that the presented framework can be used as mathematical tools which will aid to evaluate the performance of sparsely coverage-based networks in network deployment related issues in data downloading scenario.

\section{Acknowledgments}

The authors would like to express their gratitude to Professor K. Pahlavan from Worcester Polytechnic Institute (WPI) for the initiation of this work. His valuable comments helped us to develop this work.

\section{References}

[1] H. Chowdhury, J.-P. Mäkelä, and K. Pahlavan, "Traffic statistics on the coverage of WLAN-BASED music shower," in Proceedings of the IEEE 17th International Symposium on Personal, Indoor and Mobile Radio Communications (PIMRC '06), pp. 1-5, Helsinki, Finland, September 2006.

[2] B. T. Ahmed and M. C. Ramón, "Infostations for highway cigar-shaped cells," Computer Communications, vol. 32, no. 4, pp. 730-735, 2009. 
[3] T. Small and Z. J. Haas, "The shared wireless infostation model-a new ad hoc networking paradigm (or where there is a whale, there is a way)," in Proceedings of the 4th ACM International Symposium on Mobile Ad Hoc Networking \& Computing (MobiHoc '03), pp. 233-244, ACM, New York, NY, USA, 2003.

[4] A. Doufexi, E. Tameh, A. Nix, S. Armour, and A. Molina, "Hotspot wireless LANs to enhance the performance of $3 \mathrm{G}$ and beyond cellular networks," IEEE Communications Magazine, vol. 41, no. 7, pp. 58-65, 2003.

[5] J. Ott and D. Kutscher, "A disconnection-tolerant transport for drive-thru internet environments," in Proceedings of the 24th Annual Joint Conference of the IEEE Computer and Communications Societies (INFOCOM '05), vol. 3, pp. 18491862, Miami, Fla, USA, March 2005.

[6] M. L. Sichitiu and M. Kihl, "Inter-vehicle communication systems: a survey," IEEE Communications Surveys and Tutorials, vol. 10, no. 2, pp. 88-105, 2008.

[7] D. J. Goodman, J. Borras, N. B. Mandayam, and R. D. Yates, "Infostations: a new system model for data and messaging services," in Proceedings of the 47th IEEE Vehicular Technology Conference, vol. 2, pp. 969-973, May 1997.

[8] J. Ott and D. Kutscher, "Drive-thru internet: IEEE 802.lib for "automobile" users," in Proceedings of the 23rd IEEE Computer and Communications Societies (INFOCOM '04), vol. 1, pp. 362-373, Hong Kong, China, March 2004.

[9] D. Tang and M. Baker, "Analysis of a local-area wireless network," in Proceedings of the 6th Annual International Conference on Mobile Computing and Networking (MOBICOM '00), pp. 1-10, ACM, New York, NY, USA, August 2000.

[10] K. David and K. Essien, "Analysis of a campus-wide wireless network," Wireless Networks, vol. 11, no. 1-2, pp. 115-133, 2005.

[11] M. Heusse, F. Rousseau, G. Berger-Sabbatel, and A. Duda, "Performance anomaly of $802.11 \mathrm{~b}$," Proceedings of the 22nd Annual Joint Conference of the IEEE Computer and Communications Societies (INFOCOM '03), vol. 2, pp. 836-843, 2003.

[12] M. Balazinska and P. Castro, "Characterizing mobility and network usage in a corporate wireless local-area network," in Proceedings of the 1st International Conference on Mobile Systems, Applications and Services (INFOCOM '03), pp. 303316, ACM, New York, NY, USA, 2003.

[13] J. Ott and D. Kutscher, "The "Drive-thru" architecture: WLAN-based Internet access on the road," in Proceedings of the IEEE 59th Vehicular Technology Conference (VTC '04), vol. 5, pp. 2615-2622, Milan, Italy, May 2004.

[14] H. Chowdhury, J.-P. Mäkelä, and K. Pahlavan, "On the random crossing of an infostation coverage," in Proceedings of the IEEE Vehicular Technology Conference (VTC'06), vol. 1, pp. 1-5, September 2006.

[15] K. Pahlavan and A. H. Levesque, Wireless Information Networks, Wiley-Interscience, 2 nd edition, 2008.

[16] T. K. Paul and T. Ogunfunmi, "Wireless LAN comes of age: understanding the IEEE 802.11n amendment," IEEE Circuits and Systems Magazine, vol. 8, no. 1, pp. 28-54, 2008.

[17] IEEE Std 802.11n-2009, "Part 11: Wireless LAN Medium Access Control (MAC) and Physical Layer (PHY) Specifications Amendment 5," pp. c1 -502, 292009.

[18] Y. Xiao, "IEEE 802.11n: enhancements for higher throughput in wireless LANs," IEEE Wireless Communications, vol. 12, no. 6, pp. 82-91, 2005. 

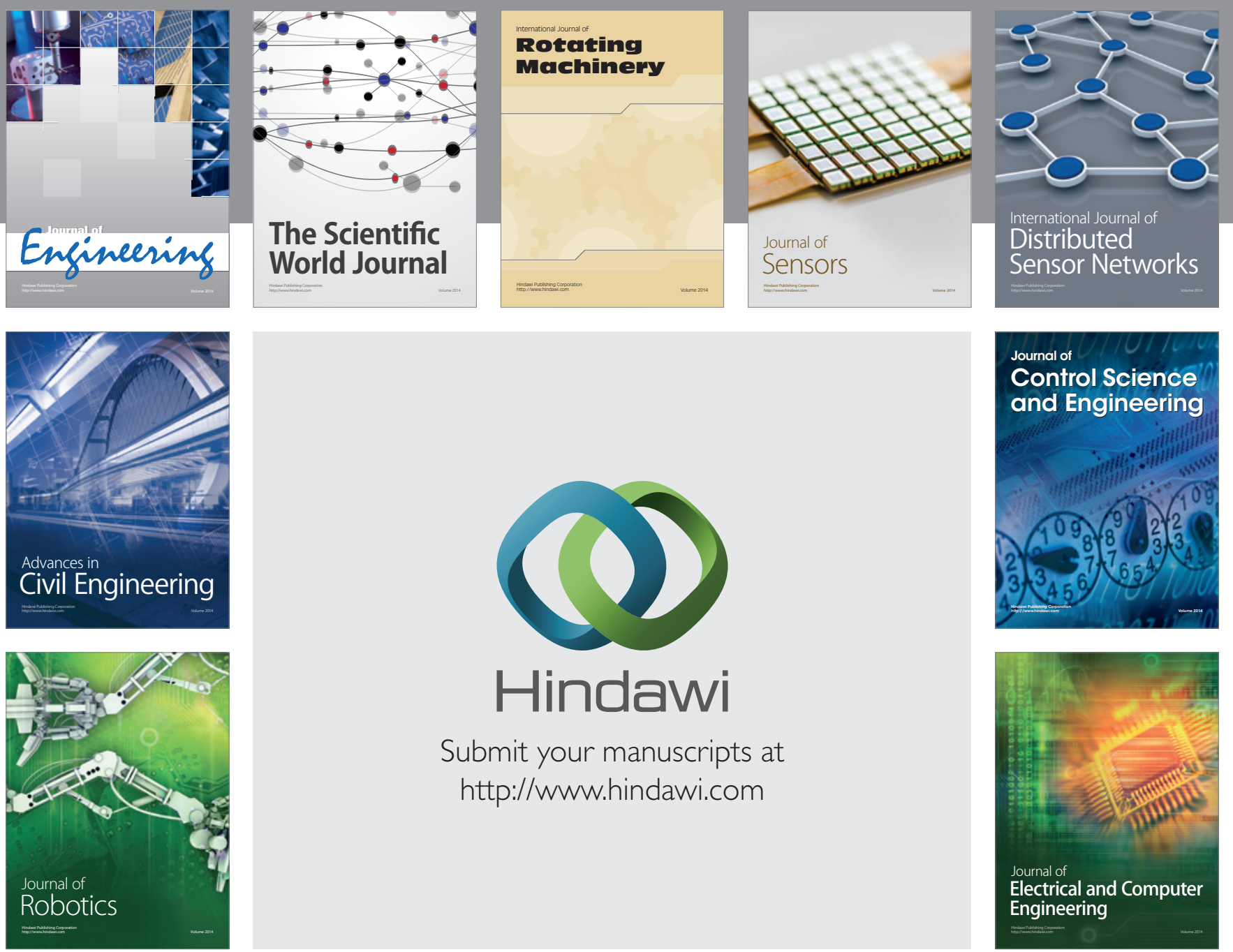

Submit your manuscripts at

http://www.hindawi.com
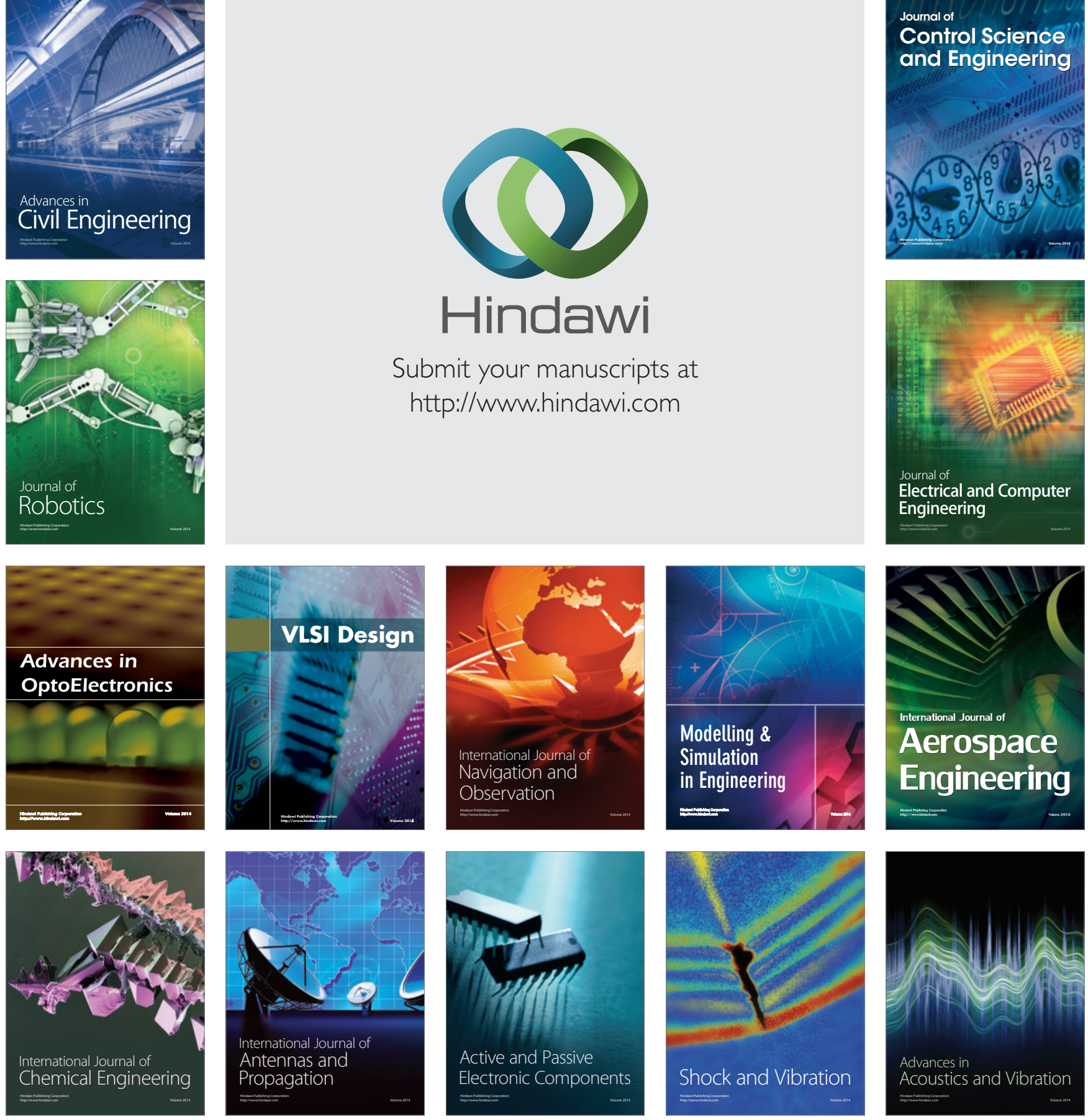